\title{
A catalogue of quasars and active nuclei: 11th edition ${ }^{\star}$
}

\author{
M.-P. Véron-Cetty and P. Véron
}

\begin{abstract}
Observatoire de Haute Provence, CNRS, 04870 Saint-Michel l'Observatoire, France
e-mail: mira.veron@oamp.fr; philippe.veron@oamp.fr
\end{abstract}

Received 25 August 2003 / Accepted 15 September 2003

\begin{abstract}
The recent release of the final installement of the $2 \mathrm{dF}$ quasar catalogue and of the first part of the Sloan catalogue, almost doubling the number of known QSOs, led us to prepare an updated version of our Catalogue of quasars and active nuclei which now contains 48921 quasars, 876 BL Lac objects and 15069 active galaxies (including 11777 Seyfert 1s). Like the tenth edition, it includes position and redshift as well as photometry $(U, B, V)$ and 6 and $11 \mathrm{~cm}$ flux densities when available. We also give a list of all known lensed and double quasars.
\end{abstract}

Key words. galaxies: quasars: general - galaxies: BL Lacertae objects: general - galaxies: active

\section{Introduction}

The first catalogue of quasars was published in 1971 by De Veny et al. It contained 202 objects. The number of known quasars has since steadily increased until the year 2000. But the release of the first part of the " $2 \mathrm{dF}$ QSO redshift survey" (Croom et al. 2001) almost doubled this number (see Table 1), The recent release of both the final installement of the $2 \mathrm{dF}$ catalogue (Croom et al. 2003) and of the first part (Abazajian et al. 2003) of the "Sloan Digital Sky Survey" (Fan et al. 1999) has again more than doubled the number of known QSOs justifying the present edition.

In this edition, containing quasars with measured redshift known to us prior to August 1st, 2003, as in the preceding editions, we do not give any information about absorption lines or X-ray properties. But we give the absolute magnitude for each object and, when available, the 11 and $6 \mathrm{~cm}$ flux densities.

This catalogue should not be used for any statistical analysis as it is not complete in any sense, except that it is, we hope, a complete survey of the literature.

\section{Description of the catalogue}

The quasars are listed in Table_QSO. A sample page is shown in Fig. 1. We have arbitrarily defined a quasar as a starlike object, or an object with a starlike nucleus, with broad emission lines, brighter than absolute magnitude $M_{B}=-23$.

Send offprint requests to: M.-P. Véron-Cetty,

e-mail: mira.veron@oamp . fr

* The catalogue (Table_QSO, Table_BL, Table_AGN and Table_reject) and the list of references are only available in electronic form at the CDS via anomymous ftp to

cdsarc.u-strasbg.fr (130.79.128.5) or via

http: //cdsweb.u-strasbg.fr/cgi-bin/qcat?]/A+A/412/399

or at the Observatoire de Haute Provence

(http://www . obs-hp. fr/).
Table 1. Increase with time of the number of known QSOs, BL Lacs and Seyfert $1 \mathrm{~s}$.

\begin{tabular}{rrrl}
\hline \hline QSO & BL Lac & Seyfert 1 & reference \\
\hline 202 & & & De Veny et al. (1971) \\
2251 & & 190 & Véron-Cetty \& Véron (1984) \\
2835 & 73 & 236 & Véron-Cetty \& Véron (1985) \\
3473 & 84 & 258 & Véron-Cetty \& Véron (1987) \\
4169 & 117 & 358 & Véron-Cetty \& Véron (1989) \\
6225 & 162 & 575 & Véron-Cetty \& Véron (1991) \\
7383 & 171 & 695 & Véron-Cetty \& Véron (1993) \\
8609 & 220 & 888 & Véron-Cetty \& Véron (1996a) \\
11358 & 357 & 1111 & Véron-Cetty \& Véron (1998) \\
13214 & 462 & 1711 & Véron-Cetty \& Véron (2000a) \\
23760 & 608 & 2765 & Véron-Cetty \& Véron (2001) \\
48921 & 876 & 11777 & Present edition \\
\hline
\end{tabular}

In Table_BL, we list all confirmed, probable or possible BL Lac objects with or without a measured redshift, without consideration of their absolute magnitude. As better spectra are becoming available, broad emission lines have been detected in a number of objects formerly classified as BL Lac; they have usually been moved to Table_QSO (Véron-Cetty \& Véron 2000b).

Table_AGN lists "active galaxies": Seyfert 1s, Seyfert 2s and Liners fainter than $M_{B}=-23$. A number of galaxies with a nuclear H II region are also included, the reason being that they have been called Seyfert in the past and later reclassified; we consider it useful to keep track of these reclassifications to avoid further confusion. Seyfert 1s have broad Balmer and other permitted lines; Seyfert 2s have Balmer and forbidden lines of the same width. Osterbrock $(1977,1981)$ has 


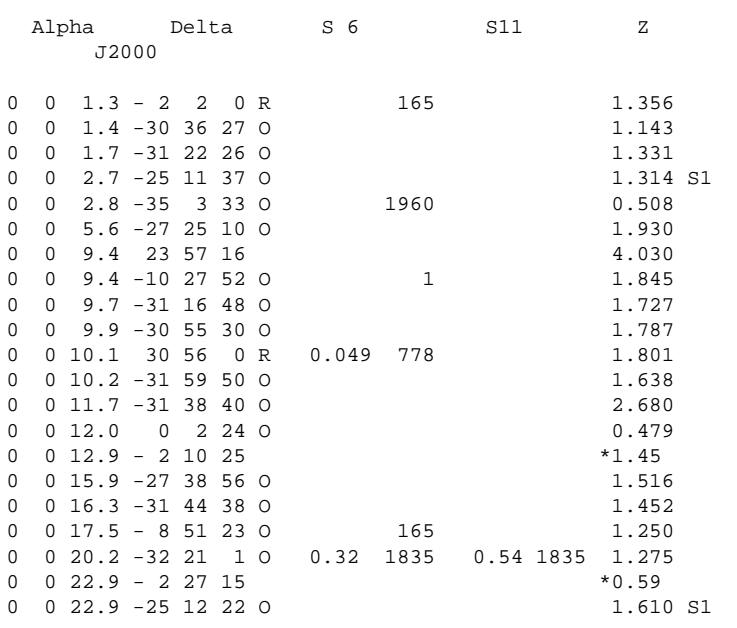

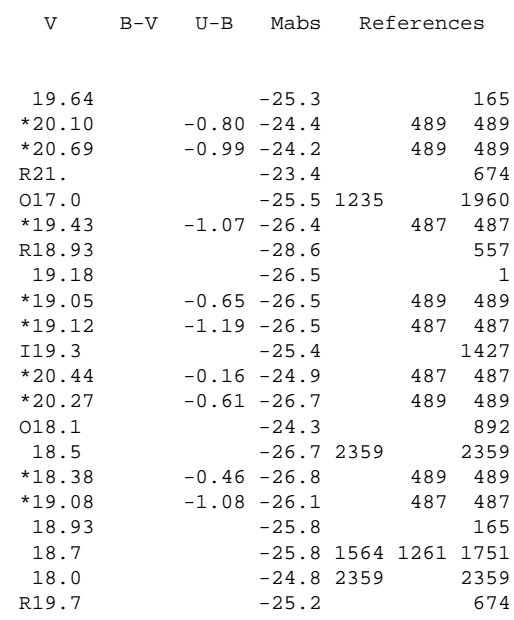

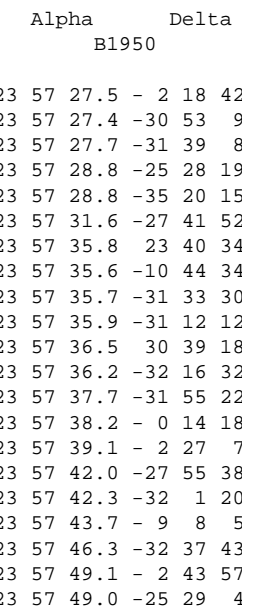

Fig. 1. Sample page of the QSO catalogue.

divided the Seyfert 1s into five subgroups: Seyfert 1.0, 1.2, 1.5, 1.8 and 1.9 on the basis of the appearance of the Balmer lines. Seyfert 1.0s are "typical" members of the class, as described by Khachikian \& Weedman $(1971,1974)$, while Seyfert 1.5s are objects intermediate between typical Seyfert $1 \mathrm{~s}$ and Seyfert $2 \mathrm{~s}$, with an easily apparent narrow $\mathrm{H} \beta$ profile superimposed on broad wings. The classes Seyfert 1.2 and 1.8 are used to describe objects with relatively weaker and stronger narrow $\mathrm{H} \beta$ components, intermediate between Seyfert 1.0 and 1.5 and Seyfert 1.5 and 2 respectively. In Seyfert 1.9, although the broad $\mathrm{H} \alpha$ emission is clearly evident, broad $\mathrm{H} \beta$ cannot be detected with certainty by mere visual inspection of the spectra. We have adopted the more quantitative classification introduced by Winkler (1992):

$\begin{array}{lll}\mathrm{S} 1.0 & 5.0 & <R \\ \mathrm{~S} 1.2 & 2.0 & <R<5.0 \\ \mathrm{~S} 1.5 & 0.333 & <R<2.0\end{array}$

S1.8 $R<0.333$ broad component visible in $\mathrm{H} \alpha$ and $\mathrm{H} \beta$ broad component visible in $\mathrm{H} \alpha$ but not in $\mathrm{H} \beta$

where $R$ is the ratio of the total $\mathrm{H} \beta$ to the [OIII] $\lambda 5007$ fluxes. Several objects have been found to show extreme spectral variability, changing from Seyfert 1.8 or 1.9 to Seyfert 1.0. In some cases these changes are consistent with changes in the reddening to the BLR while, in others, they are probably due to real changes in ionizing flux (Goodrich 1989a, 1995; Tran et al. 1992b). In some Seyfert $2 \mathrm{~s}$, a broad $\mathrm{Pa} \beta$ line has been detected, indicating the presence of a highly reddened broad line region (Goodrich et al. 1994); we call these objects S1i. A number of Seyfert 2 s have, in polarized light, the spectra of Seyfert 1s (Antonucci \& Miller 1985; Miller \& Goodrich 1990; Tran et al. 1992a); we call them S1h. Typical full widths at half-maximum of the Balmer lines in Seyfert 1s lie in the range $2000-6000 \mathrm{~km} \mathrm{~s}^{-1}$; however, there is a group of active galactic nuclei with all the properties of Seyfert $1 \mathrm{~s}$, but with unusually narrow Balmer lines (Osterbrock \& Pogge 1985;
Goodrich 1989b); they are defined as having the broad component of the Balmer lines narrower than $2000 \mathrm{~km} \mathrm{~s}^{-1}$ FWHM (Osterbrock 1987); we call them S1n. Liners (as defined by Heckman 1980) are called S3. If broad Balmer lines are observed, they are called S3b; if these broad Balmer lines are only seen in polarized light, they are called S3h. Only objects brighter than $M_{B}=-23$ appear in Table_QSO, but, clearly, some objects would move from Table_QSO to Table_AGN and vice versa if other values for $q_{0}$ and the spectral index were used or if an accurate $B$ apparent magnitude was available for all objects. The variability may have a similar effect, as well as the size of the diaphragm used for the measurement as the contribution of the underlying galaxy for weak quasars may not be negligible.

Table_reject lists the objects which once were believed to be quasars or BL lac objects and are now known to be either stars or normal galaxies.

Table_QSO contains 48921 objects, Table_BL, 876, Table_AGN, 15069 and Table_reject, 76. The catalogue is believed to contain all known quasars, BL Lac objects and Seyfert $1 \mathrm{~s}$.

Table_QSO, Table_BL and Table_AGN give:

1) Columns 1 and 2. The most common name of the object. For the meaning and the sources of the designations see Hewitt \& Burbidge (1987), Fernandez et al. (1983) and Kesteven \& Bridle (1977). For the sources discovered by the ROSAT X-ray satellite, we have used the following acronyms: RXS for the sources appearing in the All-Sky Bright Source Catalogue (Voges et al. 1999), RX for the sources in the Faint Source Catalogue (Voges et al. 2000) and 1WGA for the sources published in the WGACAT catalogue (White et al. 1994).

When the name is preceded by an *, the object has not been explicitely associated with a radio source. In fact many of these objects may have been detected by large deep radio surveys such as the FIRST (Becker et al. 1995; White et al. 1997) and NVSS (Condon et al. 1998) surveys.

2) Columns 3 to 10 . The best available J2000 optical or radio coordinates. The $\mathrm{J} 2000$ positions have been converted from the B1950 positions using the matrix given by Aoki et al. (1983). An $O$ or an $R$ following the coordinates 
means that the position is either an optical or a radio position measured with an accuracy better than one arcsec. An $A$ means that it is only an approximate position which may be wrong by several arcminutes. No reference is given for the source of the positions. The availability of the Digitized Sky Survey (DSS) allows quick measurements of the optical position of any object brighter than $\approx 19.5 \mathrm{mag}$. It has already been used to measure the position of several hundreds QSOs (Schneider et al. 1992; Bowen et al. 1994; Kirhakos et al. 1994; Véron-Cetty \& Véron 1996b). Optical positions with an accuracy better than $2^{\prime \prime}$ have also been measured for the 19369 galaxies in the Zwicky catalogue (Falco et al. 1999) and for the 12921 UGC galaxies (Cotton et al. 1999).

3) Columns 11 to 14 . The 6 and $11 \mathrm{~cm}$ flux densities (in Jy) with references to the literature. When several measurements are available we took arbitrarily one of them. When a reference is given for the $6 \mathrm{~cm}$ flux density but the value of the flux density itself is left blank and there is an * in Col. 1, only an upper limit is available and this upper limit is not much greater than $1 \mathrm{mJy}$; in case there is no* in Col. 1 , the reference refers to a detection but at a wavelength other than $6 \mathrm{~cm}$.

4) Columns 15 and 16. The redshift as published. An * in front of the redshift means that it has been estimated from a low dispersion slitless spectrum and is of lesser accuracy or even plainly wrong as the emission lines may easily be misidentified. We have given only those values which are described as probable in the original sources and not the possible values.

5) Column 17. In this column an attempt has been made to classify the objects as $\mathrm{S} 1, \mathrm{~S} 1.0, \mathrm{~S} 1.2, \mathrm{~S} 1.5, \mathrm{~S} 1.8, \mathrm{~S} 1.9, \mathrm{~S} 1 \mathrm{i}$, S1h, S1n, S2, S3, S3b, S3h, S, S? or H2. Low redshift quasars are classified as $\mathrm{S} 1$ when a good spectrum shows that they are similar to Seyfert 1 galaxies.

In Table_BL, we find in this column:

$\begin{array}{ll}\text { BL } & \text { for a confirmed BL Lac object. } \\ \text { BL? } & \text { for a probable BL Lac. } \\ \text { blank } & \text { for a possible BL Lac. } \\ ? & \text { for a questionable BL Lac } \\ \text { HP } & \text { for a Highly Polarized object. }\end{array}$

6) Columns 18 to 21 . The $V, B-V$ and $U-B$ photoelectric or photographic magnitude and colours, when available (the survey of the literature for photographic colours may be incomplete) (an $*$ in front of the magnitude indicates that the colours and the magnitude are photographic, while an $R$ or an $I$ indicates a red or an infrared magnitude). The column labelled " $V$ " gives the $V$ magnitude when $B-V$ is also given. When $B-V$ is not given, this column usually gives the $B$ magnitude, unless it is preceded by an $R$ or an $I$. Maoz et al. (1993) have measured homogeneous $V$ magnitudes for 354 QSOs with an accuracy of \pm 0.1 mag; they have been included. For a few objects, the $O$ magnitude, measured on the blue Palomar Sky Survey plates, or the UK Science Research Council SRC-J Survey plates, believed to be accurate within $\pm 0.2 \mathrm{mag}$, has been extracted from the APS database (Pennington et al. 1993). For a number of objects we give the $O$ magnitude, extracted from the USNO-A2 catalogue (Monet et al. 1996) or the Cambridge Automated Plate
Measuring Machine (APM) catalogue (Irwin et al. 1994), recalibrated by E. Flesch (private communication); these magnitudes are flagged with an $O$. The $O$ and Johnson $B$ magnitudes are related by $B-O=-(0.27 \pm 0.06) \times(B-V)$ (Evans 1989).

In the other cases, the magnitude given is an estimate as found in the original publications. These magnitudes are generally quite inaccurate and inhomogeneous; they are most often $m_{\mathrm{pg}}$ or $B$ magnitudes instead of the Johnson $V$ magnitude. Much care should be taken when using them for any purpose. Anyway, even when a photoelectric $V$ magnitude is given, it is not very meaningful as most quasars are variable. On the other hand, the colours of quasars vary little, so the listed colours should be accurate. Again, it should be noted that some of the colours listed are photographic and, therefore, less accurate; moreover, in each catalogue of photoelectric measurements, the faintest objects measured are affected by relatively large errors; this too should not be overlooked. For the galaxies, in Table_AGN, we have chosen the magnitudes and colours measured in the smallest possible diaphragm (preferentially 16 arcsec) as we are interested in the nucleus rather than in the galaxy itself.

7) Column 22. The absolute magnitude $M_{B}$ computed assuming $H_{0}=50 \mathrm{~km} \mathrm{~s}^{-1} \mathrm{Mpc}^{-1}, q_{0}=0$, and an optical spectral index $\alpha$ equal to 0.3 (defined as $S \propto v^{-\alpha}$ ) (Francis et al. 1991), as follows:

$M=m+5-5 \times \log D-k+\Delta m(z)$

where $m$ is the $B$ magnitude, $D=c / H_{0} \times A$, with $A$ the photometric distance (Terrell 1977):

$A=z\left[1+\frac{z\left(1-q_{0}\right)}{\left(1+2 q_{0} z\right)^{0.5}+1+q_{0} z}\right]$

$z$ is the redshift; $k=-2.5 \log (1+\mathrm{z})^{1-\alpha}$ is the $k$ correction, $\Delta m(z)$ is a correction to $k$ taking into account the fact that the spectrum of quasars is not strictly a power law of the form $S \propto v^{-\alpha}$, but is affected by emission lines and by the Ly $\alpha$ forest depleting the continuum to the blue of Ly $\alpha$. Assuming that the spectrum is a power law with $\alpha=0.3$ may not give the best possible estimate of the $k$ correction (Wisotzki 2000). The $R$ magnitudes have been transformed into the $B$ system by using an average $\langle B-R\rangle=0.57$ and the $I$ magnitudes by using $\langle B-I\rangle=1.1$ for low $\mathrm{z}$ QSOs. When the reference for the magnitude is $\mathrm{MaOz}$ et al. (1993), the magnitude is $V$ and we have used $\langle B-V\rangle=$ 0.40 .

8) The next three columns (23 to 25 ) give the reference for the finding chart, the photometry and the redshift respectively. In many cases, the last reference in Table_AGN is that of the classification of the object (as a Seyfert or otherwise); in these cases the redshift can usually be found in Palumbo et al. (1983).

9) The B1950 position (Cols. 26 to 32).

Since the discovery in 1979 by Walsh et al. of the first gravitationally lensed quasar, Q $0957+561$, a number of such objects (52) and of physical pairs with separation less than $10^{\prime \prime}$ (14) have been found. They are listed in Tables 2 and 3 respectively. Mortlock et al. (1999) have stressed the difficulty sometimes encountered in distinguishing lensed quasars from physical pairs. 
Table 2. Gravitationally lensed quasars. Column 1: name, Col. 2: short 1950 position, Col. 3: redshift of the quasar, Col. 4: redshift of the lens, Col. 5: separation in arcsec, Col. 6: references (see Table 3).

\begin{tabular}{|c|c|c|c|c|c|}
\hline Name & Position & $z_{\text {quasar }}$ & $z_{\text {lens }}$ & $\operatorname{sep}\left({ }^{\prime \prime}\right)$ & Ref. \\
\hline PKS 0132-097 & $0132-09$ & 2.216 & 0.764 & 0.7 & $13,18,68$ \\
\hline UM 673 & $0142-10$ & 2.719 & & 2.2 & 60 \\
\hline CTQ 414 & $0156-43$ & 1.29 & & 1.2 & 46 \\
\hline B2 $0218+35$ & $0218+35$ & 0.936 & & 0.33 & 15 \\
\hline HE 0230-2130 & $0230-21$ & 2.162 & & 2.0 & 72 \\
\hline Q J0240-343 & $0238-34$ & 1.406 & & 6.1 & 62 \\
\hline PKS 0411+05 & $0411+05$ & 2.639 & 0.958 & 2.2 & 34,64 \\
\hline HE 0435-1223 & $0435-12$ & 1.689 & & 2.6 & 73 \\
\hline HE 0512-3329 & $0512-33$ & 1.565 & 0.931 & 0.6 & 12 \\
\hline B $0712+472$ & $0712+47$ & 1.339 & & 1.27 & 8 \\
\hline MG $0751+2716$ & $0748+27$ & 3.200 & 0.350 & & 64 \\
\hline HS $0810+25$ & $0810+25$ & 1.500 & & 0.25 & 55 \\
\hline HS $0818+1227$ & $0818+12$ & 3.115 & & 2.1 & 16 \\
\hline CLASS B0827+525 & $0827+52$ & 2.064 & & 2.8 & 31 \\
\hline APM $08279+5255$ & $0827+52$ & 3.87 & & 0.4 & 35 \\
\hline SDSS $09035+5028$ & $0900+50$ & 3.584 & 0.388 & 2.8 & 28 \\
\hline RX J0911.4+0551 & $0908+06$ & 2.800 & & 0.8 & 1 \\
\hline SBS 0909+532 & $0909+53$ & 1.377 & 0.830 & 1.11 & 30,39 \\
\hline 1WGA J09212+4528 & $0917+45$ & 1.66 & 0.31 & 6.93 & 51 \\
\hline SDSSp J09249+0219 & $0922+02$ & 1.524 & & 1.8 & 26 \\
\hline FBQS J0951+2635 & $0948+26$ & 1.24 & & 1.1 & 56 \\
\hline BRI 0952-01 & $0952-01$ & 4.43 & & 0.95 & 43 \\
\hline Q $0957+561$ & $0957+56$ & 1.414 & 0.355 & 6.1 & 11,65 \\
\hline FIRST J10044+1229 & $1001+12$ & 2.65 & & 1.54 & 32 \\
\hline Q 1009-0252 & $1009-02$ & 2.74 & & 1.55 & 21 \\
\hline J 13.03 & $1015-20$ & 2.55 & & 0.84 & 61 \\
\hline IRAS F10214+4724 & $1021+47$ & 2.286 & & & 57 \\
\hline B $1030+074$ & $1030+07$ & 1.535 & & 1.56 & 8 \\
\hline HE $1104-1805$ & $1104-18$ & 2.303 & 0.729 & 3.0 & 37,70 \\
\hline PG $1115+080$ & $1115+08$ & 1.722 & 0.311 & 2.3 & 63,66 \\
\hline UM 425 & $1120+01$ & 1.465 & & 6.5 & 44 \\
\hline 1RXS J11319-1231 & $1129-12$ & 0.658 & 0.295 & 4.2 & 58 \\
\hline TEX 1152+199 & $1152+19$ & 1.019 & 0.439 & 1.6 & 52 \\
\hline Q 1208+1011 & $1208+10$ & 3.803 & & 0.45 & 41 \\
\hline 87 GB $1359+1527$ & $1359+15$ & 3.235 & & 1.7 & 52 \\
\hline H $1413+117$ & $1413+11$ & 2.546 & & 1.4 & 40 \\
\hline HST J14176+5226 & $1415+52$ & 3.4 & & 3.2 & 5 \\
\hline B $1422+231$ & $1422+23$ & 3.62 & 0.339 & 1.3 & 53,63 \\
\hline SBS $1520+530$ & $1520+53$ & 1.855 & 0.717 & 1.6 & 3,4 \\
\hline Q $1600+434$ & $1600+43$ & 1.61 & & 1.38 & 27 \\
\hline FIRST J1633+3134 & $1631+31$ & 1.516 & & 0.66 & 48 \\
\hline PMN J1632-0033 & $1630-00$ & 3.424 & & 1.46 & 69 \\
\hline Q $1634.9+26.7$ & $1634+26$ & 1.961 & & 3.8 & 59 \\
\hline SDSSp J16507+4251 & $1649+42$ & 1.541 & & 1.16 & 49 \\
\hline MC 1830-211 & $1830-21$ & 2.507 & 0.885 & 0.60 & 36,38 \\
\hline TEX 1835-345 & $1835-34$ & 2.78 & & 1.0 & 67 \\
\hline MG 2019+1127 & $2016+11$ & 3.273 & & 3.4 & 33 \\
\hline 87 GB $20451+2632$ & $2045+26$ & 1.28 & 0.867 & 1.9 & 9 \\
\hline Q 2138-431 & $2138-43$ & 1.641 & & 4.5 & 19 \\
\hline HE 2149-2745 & $2149-27$ & 2.033 & & 1.7 & 71 \\
\hline Q 2237+0305 & $2237+03$ & 1.695 & 0.039 & 1.8 & 24 \\
\hline Q 2345+007 & $2345+00$ & 2.15 & & 7.1 & 54 \\
\hline
\end{tabular}

Acknowledgements. This research has made use of the APS catalogue of POSS I database which is supported by the National Science
Foundation, the National Aeronautics and Space Administration, and the University of Minnesota. We are very grateful to E. Flesch 
Table 3. Quasar pairs. Column 1: name, Col. 2: short 1950 position, Col. 3: redshift of the quasar, Col. 4: separation in $\operatorname{arcsec}$, Col. 5: references.

\begin{tabular}{lllcr}
\hline \hline Name & Position & $z$ & sep $\left(^{\prime \prime}\right)$ & Ref. \\
\hline LBQS 0015+02 & $0015+02$ & 2.469 & 2.2 & 25 \\
Q 0023+171 & $0023+17$ & 0.945 & 4.8 & 23 \\
CT 344 & $0103-27$ & 0.848 & 0.3 & 29 \\
PHL 1222 & $0151+04$ & 1.910 & 3.3 & 45 \\
CTQ 839 & $0250-33$ & 2.24 & 2.1 & 47 \\
Q 1145-071 & $1145-07$ & 1.34 & 4.2 & 7 \\
HS 1216+5032 & $1216+50$ & 1.450 & 8.9 & 17 \\
RRS IV 26,27 & $1343+26$ & 2.030 & 9.5 & 6 \\
Q 1429-008 & $1429-00$ & 2.076 & 5.1 & 10,20 \\
RX J16290+3724 & $1627+37$ & 0.923 & 4.3 & 42 \\
Q J1643+31 & $1641+32$ & 0.586 & 2.3 & 2 \\
Q 2153-2056 & $2153-20$ & 1.845 & 7.8 & 22 \\
MGC 2214+3550 & $2212+35$ & 0.877 & 3.0 & 50 \\
SDSSp J23365-0107 & $2334-01$ & 1.285 & 1.7 & 14 \\
\hline
\end{tabular}

References: (1) Bade et al. (1997); (2) Brotherton et al. (1999); (3) Burud et al. (2002); (4) Chavushyan et al. (1997); (5) Crampton et al. (1996); (6) Crotts et al. (1994); (7) Djorgovski et al. (1987); (8) Fassnacht \& Cohen (1998); (9) Fassnacht et al. (1999); (10) Faure et al. (2003); (11) Garrett et al. (1992); (12) Gregg et al. (2000); (13) Gregg et al. (2001); (14) Gregg et al. (2002); (15) Grundahl \& Hjorth (1995); (16) Hagen \& Reimers (2000); (17) Hagen et al. (1996); (18) Hall et al. (2002); (19) Hawkins et al. (1997); (20) Hewett et al. (1989); (21) Hewett et al. (1994); (22) Hewett et al. (1998); (23) Hewitt et al. (1987); (24) Huchra et al. (1985); (25) Impey et al. (2002); (26) Inada et al. (2003); (27) Jackson et al. (1995); (28) Johnston et al. (2003). (29) Junkkarinen et al. (2001); (30) Kochanek et al. (1997); (31) Koopmans et al. (2000); (32) Lacy et al. (2002); (33) Lawrence et al. (1984); (34) Lawrence et al. (1995); (35) Ledoux et al. (1998); (36) Lidman et al. (1999); (37) Lidman et al. (2000); (38) Lowell et al. (1998); (39) Lubin et al. (2000); (40) Magain et al. (1988); (41) Magain et al. (1992); (42) Mason et al. (2000); (43) McMahon et al. (1992); (44) Meylan \& Djorgovski (1989); (45) Meylan et al. (1990); (46) Morgan et al. (1999); (47) Morgan et al. (2000); (48) Morgan et al. (2001); (49) Morgan et al. (2003); (50) Muñoz et al. (1998); (51) Muñoz et al. (2001); (52) Myers et al. (1999); (53) Patnaïk et al. (1992); (54) Pello et al. (1996); (55) Reimers et al. (2002); (56) Schechter et al. (1998); (57) Serjeant et al. (1995); (58) Sluse et al. (2003); (59) Steidel \& Sargent (1991); (60) Surdej et al. (1987); (61) Surdej et al. (1997); (62) Tinney (1995); (63) Tonry (1998); (64) Tonry \& Kochanek (1999); (65) Tytler \& Fan (1992); (66) Weymann et al. (1980); (67) Winn et al. (2000); (68) Winn et al. (2001); (69) Winn et al. (2002); (70) Wisotzki et al. (1993); (71) Wisotzki et al. (1996); (72) Wisotzki et al. (1999); (73) Wisotzki et al. (2002).

and F. Ochsenbein for checking and improving the catalogue and we thank R. Monella for having brought to our attention a number of errors and omissions in previous editions of this catalogue.

\section{References}

Abazajian, K., Adelman-McCarthy, J. K., Agueras, M. A., et al. 2003, $\mathrm{AJ}$, in press [astro-ph/0305492]

Antonucci, R. R. J., \& Miller, J. S. 1985, ApJ, 297, 621

Aoki, S., Sôma, M., Kinoshita, H., \& Inoue, K. 1983, A\&A, 128, 263
Becker, R. H., White, R. L., \& Helphand, D. J. 1995, ApJ, 450, 559

Burud, I., Hjorth, J., Courbin, F., et al. 2002, A\&A, 391, 481

Condon, J. J., Cotton, W. D., Greisen, E. W., et al. 1998, AJ, 115, 1693

Cotton, W. D., Condon, J. J., \& Arbizzani, E. 1999, ApJS, 125, 409

Croom, S. M., Smith, R. J., Boyle, B. J., et al. 2001, MNRAS, 322, L29

Croom, S. M., Smith, R. J., Boyle, B. J., et al. 2003, MNRAS, in press De Veny, J. B., Osborn, W. H., \& Janes, K. 1971, PASP, 83, 611

Evans, D. W. 1989, A\&AS, 78, 249

Falco, E. F., Kurtz, M. J., Geller, M. J., et al. 1999, PASP, 111, 438

Fan, X., Strauss, M. A., Schneider, D. P., et al. 1999, AJ, 118, 1

Faure, C., Alloin, D., Gras, S., et al. 2003, A\&A, 405, 415

Fernandez, A., Lortet, M.-C., \& Spite, F. 1983, A\&AS, 52, 4

Francis, P. J., Hewett, P. C., Foltz, C. B., et al. 1991, ApJ, 373, 465

Goodrich, R. W. 1989a, ApJ, 340, 190

Goodrich, R. W. 1989b, ApJ, 342, 224

Goodrich, R. W. 1995, ApJ, 440, 141

Goodrich, R. W., Veilleux, S., \& Hill, G. J. 1994, ApJ, 422, 521

Hall, P. B., Richards, G. T., York, D. G., et al. 2002, ApJ, 575, L51

Heckman, T. M. 1980, A\&A, 87, 152

Irwin, M., Maddox, S., \& McMahon, R. 1994, Spectrum, 2, 14

Kesteven, M. J. L., \& Bridle, A. H. 1977, J. Roy. Astron. Soc. Canada, 71,21

Khachikian, E. E., \& Weedman, D. W. 1971, Astrophys., 7, 231

Khachikian, E. E., \& Weedman, D. W. 1974, ApJ, 192, 581

Miller, J. S., \& Goodrich, R. W. 1990, ApJ, 355, 456

Monet, D., Bird, A., Canzian, B., et al. 1996, USNO-A2.0, US Naval Observatory, Washington DC

Mortlock, D. J., Webster, R. L., \& Francis, P. J. 1999, MNRAS, 309, 836

Osterbrock, D. E. 1977, ApJ, 215, 733

Osterbrock, D. E. 1981, ApJ, 249, 462

Osterbrock, D. E. 1987, Lecture Notes in Physics, 307, 1

Osterbrock, D. E., \& Pogge, R. W. 1985, ApJ, 297, 166

Pennington, R. L., Humphreys, R. M., Odewahn, S. C., Zumach, W., \& Thurmes, P. M. 1993, PASP, 105, 521

Reimers, D., Hagen, H.-J., Baade, R., Lopez, S., \& Tytler, D. 2002, A\&A, 382, L26

Schneider, D. P., Bahcall, J. N., Saxe, D. H., et al. 1992, PASP, 104, 678

Terrell, J. 1977, Am. J. Phys., 45, 869

Tran, H. D., Miller, J. S., \& Kay, L. E. 1992a, ApJ, 397, 452

Tran, H. D., Osterbrock, D. E., \& Martel, A. 1992b, AJ, 104, 2072

Véron-Cetty, M.-P., \& Véron, P. 1984, ESO Scientific Report, No. 1

Véron-Cetty, M.-P., \& Véron, P. 1985, ESO Scientific Report, No. 4

Véron-Cetty, M.-P., \& Véron, P. 1987, ESO Scientific Report, No. 5

Véron-Cetty, M.-P., \& Véron, P. 1989, ESO Scientific Report, No. 7

Véron-Cetty, M.-P., \& Véron, P. 1991, ESO Scientific Report, No. 10

Véron-Cetty, M.-P., \& Véron, P. 1993, ESO Scientific Report, No. 13

Véron-Cetty, M.-P., \& Véron, P. 1996a, ESO Scientific Report, No. 17 Véron-Cetty, M.-P., \& Véron, P. 1996b, A\&AS, 115, 97

Véron-Cetty, M.-P., \& Véron, P. 1998, ESO Scientific Report, No. 18 Véron-Cetty, M.-P., \& Véron, P. 2000a, ESO Scientific Report, No. 19 Véron-Cetty, M.-P., \& Véron, P. 2000b, A\&AR, 10, 81

Véron-Cetty, M.-P., \& Véron, P. 2001, A\&A, 374, 92

Voges, W., Aschenbach, B., Boller, T., et al. 1999, A\&A, 349, 389

Voges, W., Aschenbach, B., Boller, T., et al. 2000, IAU Circ., 7432

Walsh, D., Carswell, R. F., \& Weymann, R. J. 1979, Nature, 279, 381

White, N. E., Giommi, P., \& Angelini, L. 1994, IAU Circ., 6100

White, R. L., Becker, R. H., Helphand, D. J., \& Gregg, M. 1997, ApJ, 475,479

Winkler, H. 1992, MNRAS, 257, 677

Winn, J. N., Lovell, J. E. J., Chen, H.-W., et al. 2001, ApJ, 564, 143

Wisotzki, L. 2000, A\&A, 353, 861 